\title{
A Theoretical Study of Acrylonitrile Adsorption on $\mathrm{Si}(001)$
}

\author{
F. de León-Pérez*, R. Miotto ${ }^{\dagger}$, and A. C. Ferraz \\ * Inst. de Física, USP, Caixa Postal 66318, CEP 05315-970, São Paulo, SP, Brazil \\ ${ }^{\dagger}$ Inst. de Física, UnB, Caixa Postal 04455, CEP 70919-970, Brasilia, DF, Brazil
}

Received on 31 March, 2003

\begin{abstract}
The present work is a comparative study of possible adsorption structures of the conjugated molecule acrylonitrile on $S i(001)$ employing the state of the art pseudopotential method, within a generalized gradient approximation to the density functional theory. In the recent literature it is proposed the interaction of acrylonitrile with $S i(001)$ through a cycloaddition reaction of the cyano group, in competition with the bounding of the two outer atoms of the molecule skeleton with the Si dimer in cross-dimer and cross-trench geometries; between other geometries like which correspond the reaction of the $C=C$ bond with a Si dimer. Starting from a large number of configurations our calculations favor the planar cycloaddition through the terminal $\mathrm{N}$ and $\mathrm{C}$ atoms on the Si dimer. In this way we explain the electronic and vibrational features obtained experimentally.
\end{abstract}

\section{Introduction}

The adsorption of organic molecules on semiconductor surfaces, in particular small hydrocarbons on $\mathrm{Si}(001)$, deserved in the past few years a great deal of effort towards the surface modification and functionalization for potential applications in semiconductor and microelectronic technologies. Acrylonitrile $\left(\mathrm{CH}_{2}=\mathrm{CH}-\mathrm{C} \equiv \mathrm{N}\right)$ is a molecule with two of the most important building blocks for many organic molecules: the vinyl and cyano groups, and has a rich chemistry (for an extensive discussion see [1] and references therein). The absorption of acrylonitrile on $\mathrm{Si}(001)$ has been recently studied both from the experimental $[1,2]$ and theoretical points of view [1]. While Tao et al [1], from a HighResolution Electron Energy Loss (HREEL) study of Si(001) exposed to acrylonitrile at $110 \mathrm{~K}$ and first-principles calculations of two possible absorption geometries, concluded that the absence of the $C \equiv N$ peak with the concurrent emergence of the $C=N$ peak is the direct evidence for the participation of the cyano group in the interaction with surface silicon dimers; Bournel et al [2], from a synchroton Photoemission Spectroscopy (PES) study and their Near Edge $\mathrm{X}$-ray Absorption Fine Structure (NEXAFS) $N$ and $C \mathrm{~K}$ edges spectra, showed that their results are not in agreement with the above proposed mechanism. They observed features corresponding to both fragments of the molecule and to the presence of $\mathrm{Si}-\mathrm{C}$ and $\mathrm{Si}-\mathrm{N}$ bonds, and also concluded that a large fraction of this molecule (of $C_{s}$ symmetry in the gas phase) is absorbed with the molecular plane parallel to the substrate plane. They suggested that only a $C N$ axis "tilted" with respect to the silicon surface could explain the unpolarized nature of the observed $\pi^{*}(2)$ transition [2].

In order to determine the most probable atomic geometry, we modeled the $\mathrm{Si}(001)$ surface in a supercell geometry, with an atomic slab of six Si layers and a vacuum region equivalent to eight atomic layers. On the top side of the slab we placed the acrylonitrile molecule in different configurations. The numerical computations are performed in the framework of density functional theory. The valenceelectron-ion interaction is treated by ab initio normconserving pseudopotentials. In particular, the pseudopotentials for $\mathrm{Si}, \mathrm{H}, \mathrm{C}$, and $\mathrm{N}$ were derived by using the scheme of Troullier and Martins [3]. The electron-electron exchangecorrelation interactions were considered by using the generalized gradient approximation (GGA) [4] of density functional theory. As for the surface calculations, the single particle orbitals were expressed in a plane-wave basis up to the kinetic energy of $35 \mathrm{Ry}$. For the Brillouin-zone summation, four special $\mathbf{k}$ points were used for surface calculations. The atoms were assumed to be in their fully relaxed positions when the forces acting on the ions were smaller than $0.005 \mathrm{eV} / \AA$. The relaxed adsorption geometries were used to calculate the zone-center vibrational modes within the frozen-phonon scheme [5]. For setting up the dynamical problem we considered only the adsorbed system (acrylonitrile molecule) and the Si dimer atoms.

\section{Results}

For bulk silicon our first-principles calculations produced $5.42 \AA$ for the equilibrium lattice constant $\left(a_{0}\right)$, in good agreement with the experimental value $(5.43 \AA)$ presented in Ref. [6]. The calculated theoretical lattice constant obtained for the bulk silicon is used in surface calculations. The clean $\mathrm{Si}(001)-(2 \times 1)$ surface is characterized by a tilted $\mathrm{Si}-\mathrm{Si}$ dimer, i.e. one dimer component is at higher position than the other. The tilting of the dimer allows charge transfer from the "down" atom (which becomes more planar or $s p^{2}$ bonded) to the "up" atom (which becomes nearly pyramidal or $s^{2} p^{3}$ bonded). Thus, the "down" $\mathrm{Si}$ atom dimer is electron deficient, while the "up" Si atom is electron rich. Our calcu- 
lations support this model: the Si-Si dimer is found to have a bond length of $2.34 \AA$ and a vertical buckling of $0.73 \AA$, indicating a tilt angle of $17.8^{\circ}$. The calculated bond lengths and angles for the acrylonitrile molecule (we label the atoms according to Ref. [7]) are $C^{1}-C^{2}=1.34 \AA, C^{2}-C^{3}=1.42$ $\AA, C^{3}-N=1.166 \AA, C^{1}-H^{1}=1.09 \AA, C^{1}-H^{2}=1.10$ $\AA, C^{2}-H^{3}=1.10 \AA, C^{1}-C^{2}-C^{3}=120.19^{\circ}$, $C^{1}-C^{2}-H^{3}=122.44^{\circ}, C^{2}-C^{3}-N=178.57^{\circ}$, $H^{1}-C^{1}-C^{2}=121.61^{\circ}, H^{2}-C^{1}-C^{2}=120.58^{\circ}$, $H^{3}-C^{2}-C^{3}=117.37^{\circ}$, and $H^{1}-C^{1}-H^{2}=117.81^{\circ}$. All values are in good agreement with available experimental data [7].

The calculated vibrational frequencies for acrylonitrile molecule are given in Table I. Experimental values are also shown for comparison. The largest relative deviation is 5.5 $\%$ for the $\mathrm{CH}_{2}$ asymmetric stretch mode, in good agreement with the known accuracy of frozen phonon calculations $(<10 \%)$. For the sake of clarity we limit ourself to the modes relevant for the present study (the topmost modes).
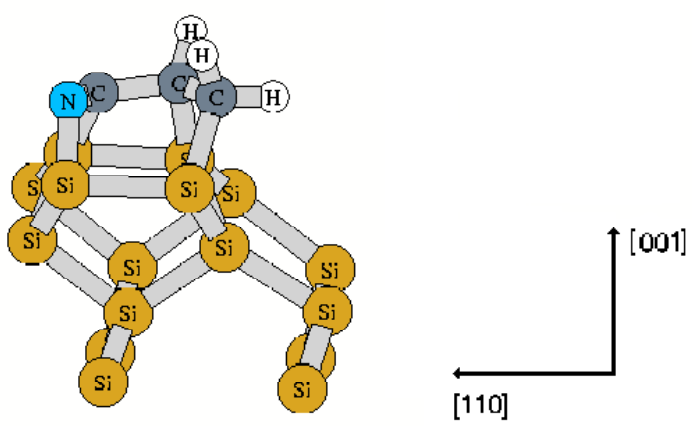

Figure 1. Schematic view of the planar cycloaddition through the two terminal $\mathrm{N}$ and $\mathrm{C}$ atoms of the acrylonitrile molecule on the $\mathrm{Si}(001)-(2 \times 2)$ surface.

Our first-principles calculations indicate that the most favorable geometry for absorption is the cycloaddition through the two terminals $\mathrm{C}$ and $\mathrm{N}$ atoms in the configuration that the plane of the molecule is parallel to the substrate plane (see Fig. 1). The relative stability of this mode is 0.12 , $0.29,0.34$, and $1.08 \mathrm{eV}$ per $(2 \times 2)$ unit cell when compared to the diagonal cross-dimer, vertical cycloaddition of the two terminal $\mathrm{C}$ and $\mathrm{N}$ atoms, cycloaddition through the two carbon atoms of the vinyl group, and cycloaddition through the cyano group structures, respectively. That agrees with the experimental findings of Bournel et al relating planar absorption geometry and the presence of $S i-C$ and $S i-N$ bonds. In the most stable structure both $\mathrm{Si}$ dimers are no more buckled but almost planar, and with a relative elongation of about $1.8 \%$ and $-0.3 \%$ for the absorption dimer and the next nearest dimer respectively. An interaction to the next nearest neighbour dimer is found $\left(C^{3}-S i\right.$ (up)= $2.01 \AA$ and the $C^{3}-S i($ down $)=2.13 \AA$ ), and in this case the coverage tends to 1 . The cycloaddition through both the $N$ atom of the cyano and the $C^{3}$ of the vinyl group with the molecular plane perpendicular to the substrate could also be possible after complete dissociation, for an activation bar- rier of $0.29 \mathrm{eV}$ corresponding to a thermal activation in the range $97-145 \mathrm{~K}$ (theses values are estimated from the phenomenological Arrhenius approach). That corresponds to the experimental situation setup by Tao et al [1]. In this case a coverage of 0.5 obtained by these authors (unfortunately Bournel et al could not report a coverage value) is possible. On the other hand, the cycloaddition through the two carbon atoms of the vinyl group (activation barrier of $0.34 \mathrm{eV}$ and related thermal activation in the range 114-171 K) shows a tilted $C=N$ bond, expected from the results of Bournel and co-workers, obtained at room temperature.

The vibrational eigenvalues for two structures of interest are found in Table I. Related to the isolated molecule, both the planar and vertical cycloaddition through the terminals $\mathrm{C}$ and $\mathrm{N}$ atoms structures ((c)[v] and (c)[p] in Table I) retain the $\mathrm{CH}_{2}$ asymmetric stretch and symmetric stretch modes, and the $\mathrm{CH}$ stretch mode (though in the vertical (c) case the $\mathrm{CH}_{2}$ asymmetric stretch and the $\mathrm{CH}$ stretch mode interchange each other). Note that through the rehybridization of the atoms of the vinyl group these modes remain almost constant, in contradiction with the opinion of Tao et al [1]. But the $C \equiv N$ and $C=C$ symmetric stretch modes behave in a different way in both structures. For planar (c) absorption the $C \equiv N$ mode led to a $C=N$ mode close to the $C=C$ mode of the isolate molecule. That is similar to the results of [1] though in our case it was not necessary a cycloaddition of the cyano group to explain this feature. On the other hand, the $C=C$ symmetric stretch mode disappears (contrary to [1] where this mode remains unchanged, or at least the spectral peak positions are the same). For the vertical (c) absorption both modes remain with about the same character of the isolated molecule. In the diagonal cross-dimer structure (not shown in Table I) a situation similar to (c)[v] is found. Neither any individual absorption geometry nor the simultaneous presence of different structures agree with all the asignements given by Tao et al in their HREEL spectra, though we explain the main results of their experiment.

Table I. Acrylonitrile vibrational modes (frequencies in $\mathrm{cm}^{-1}$ ) for both the isolated molecule and the molecule absorbed both vertical and planar through the terminal atoms $\mathrm{C}$ and $\mathrm{N}$ on the $\mathrm{Si}(001)$ surface (vertical(c)[v] and planar (c)[p] modes, respectively). Only the modes relevant to this work are reported. Experimental results for the isolated molecule are taken from [8].

\begin{tabular}{lcccc}
\hline structure & \multicolumn{2}{c}{ Molecule } & \multicolumn{2}{c}{$\mathrm{Si}(001)$} \\
\hline modes & this work & $\exp$ & (c) $[\mathrm{v}]$ & $(\mathrm{c})[\mathrm{p}]$ \\
\hline$=\mathrm{CH}_{2}$ asym. stretch & 3296 & 3125 & 3163 & 3211 \\
$=\mathrm{CH}$ stretch & 3157 & 3078 & 3254 & 3102 \\
$=\mathrm{CH}_{2}$ sym. stretch & 3104 & 3042 & 2996 & 3037 \\
$\mathrm{C} \equiv \mathrm{N}$ stretch & 2354 & 2239 & 2244 & 1751 \\
$\mathrm{C}=\mathrm{C}$ sym. stretch & 1570 & 1615 & 1335 & \\
\hline
\end{tabular}

The most striking feature comparing with the results of Tao et al is that, in our calculations, the total energy for the cycloaddition of the cyano group is about $18 \mathrm{kcal} \cdot \mathrm{mol}^{-1}$ $(1.08 \mathrm{eV})$ above the total energy of vertical cycloaddition 
by the two terminal atoms, while in [1] the former total energy is $\sim 3-6 \mathrm{kcal} \cdot \mathrm{mol}^{-1}$ below the second one. Unfortunately these authors did not report the parameter values of their structures for comparison. They only commented that the $C^{2} C^{1} N$ angle is nearly $180^{\circ}$ while in our calculations this angle decreases to $163^{\circ}$, and the $C^{1} C^{2} C^{3}$ angle reduces to $111^{\circ}$ in order to diminish the strain in the molecule (we obtain for the isolated molecule the values $179^{\circ}$ and $120^{\circ}$ respectively). Finally, its worthy pointing out that the differences between the bindig energies of both structures suggest that the cycloaddition via the cyano group is unlikely to be observed at room temperature.

\section{Conclusion}

Using a first-principles pseudopotential technique we have investigated 10 possible structures for the absorption of acrylonitrile on the $\mathrm{Si}(001)$ surface. At low temperatures, the cycloaddition of the two terminal $\mathrm{C}$ and $\mathrm{N}$ atoms is found to be the most stable structure. The relative stability of this mode is $0.12,0.29,0.34$, and $1.08 \mathrm{eV}$ per $(2 \times 2)$ unit cell when compared to the diagonal cross-dimer, vertical cycloaddition of the two terminal $\mathrm{C}$ and $\mathrm{N}$ atoms, cycloaddition through the two carbon atoms of the group, and cycloaddition through the cyano group structures, respectively. The absorbed system is locally (i.e. at the absorbed site) characterized by symmetric and slightly elongated $\mathrm{Si}$ Si dimers. Our total energy calculations further suggest that other metastable configurations, like the diagonal crossdimer and the vertical cycloaddition of the two terminal $\mathrm{C}$ and $\mathrm{N}$ atoms, are also possible. Recent experimental studies are explained with our theoretical model.

\section{Acknowledgments}

The authors acknowledge financial support from the brazilian agencies FAPESP, CNPq, and DPP-UnB.

\section{References}

[1] F. Tao, W. S. Sim, G. Q. Xu, and M. H. Qiao, J. Am. Chem. Soc 123, 9397 (2001).

[2] F. Bournel, J.-J. Gallet, S. Kubsky, G. Dufour, F. Rochet, M. Simeoni, and F. Sirotti, Surf. Sci. 513, 37 (2002).

[3] N. Troullier and J. L. Martins, Phys. Rev. B 43, 1993 (1991).

[4] J. P. Perdew, K. Burke, and M. Ernzerhof, Phys. Rev. Lett. 77, 3865 (1996).

[5] G. P. Srivastava, The Physics of Phonons (Adam Hilger, Bristol, 1990).

[6] Handbook of Chemistry and Physics (Chemical Rubber, Boca Raton, 1995).

[7] Landolt-Börnstein Numerical Data and functional Relationships in Science and Technology, Vol. 7, edited by: K.H. Hellwege and A. M. Hellwege, (Springer-Verlag, Berlin, 1976).

[8] X. Crispin, c. Bureau, V. M. Geskin, R. Lazzaroni, W. R. Salaneck, and J. L. Breédas, J. Chem. Phys. 111, 3237 (1999); NIST Chemistry WebBook (http://webbook.nist.gov/chemistry). 